\title{
Continuity of care: influence of general practitioners' knowledge about their patients on use of resources in consultations
}

\author{
Per Hjortdahl, Christian Fr Borchgrevink
}

\begin{abstract}
Objective-To examine the relation between general practitioners' knowledge about their patients and the use of resources in consultations.

Design-A cross sectional evaluation of consultations.

Setting and subjects - A representative sample of 133 Norwegian general practitioners were each asked to record 30 consecutive consultations. 131 did so, and of 3990 possible registrations, $3918(98 \%)$ were evaluated.
\end{abstract}

Main outcome measures-The influence, as assessed by the doctor, of accumulated knowledge on the use of laboratory tests, expectant management, prescriptions, sickness certification, referrals, and time spent in the consultation.

Results-Accumulated knowledge was a substantial factor in saving time, especially in consultations with children, the elderly, patients with psychosocial problems, and those with chronic diseases. It also influenced the overall use of laboratory tests, expectant management, sickness certification, and referrals, and to a lesser degree the use of medication.

Conclusion-The findings imply strong but complex associations between accumulated knowledge and the use of resources in the consultation.

\section{Introduction}

Continuity of care, defined as medical care over time provided by one health care worker regardless of the presence of any specific disease, is believed to enhance the quality of medical care. ${ }^{1}$ One of several ways continuity may exert such an influence is through the doctor's accumulated knowledge of the patient. This knowledge may in turn influence the use of resources, such as time spent with the patient and diagnostic and therapeutic measures.

The influence of continuity of care on the use of resources is important both at the individual level ${ }^{2}$ and more generally. ${ }^{3+}$ The doctor's knowledge of a patient may work both ways. When the patient is unknown the clinician may be reluctant to prescribe antidepressant drugs though indicated, or may want to perform a few extra laboratory tests. On the other hand, when a familiar patient complains of recurrent stomach pains the doctor may use expectant management ("wait and see") instead of immediate referral. Even though the direct cost of a consultation in general practice is modest, each consultation may generate large expenses in the way of prescriptions, laboratory tests, referrals, and sickness certification. The annual number of consultations in general practice is so high that this use of resources has an effect on costs within the whole health care system.

Our aim was to evaluate the influence of doctors' accumulated knowledge about patients on the use of resources in the consultation.

\section{Subjects and methods}

The study took place during the spring of 1987. A random sample of general practitioners was drawn from the membership list of the Norwegian Medical Association, which includes $95 \%$ of all doctors in the country. One hundred and thirty three practitioners each agreed to record 30 consecutive consultations. Both scheduled and unscheduled consultations with patients of all ages were included. Telephone contacts, house calls, and organised preventive work such as well baby clinics were excluded. The doctors completed their recordings within two to five days. As patients who made two or more visits within the study period were recorded only once, the number of records equalled the number of different patients seen.

Immediately after each consultation the doctor completed a two page questionnaire related to continuity of care, knowledge about the patient, and use of resources in the consultation. The validity of the questionnaire was tested by an expert panel of five doctors and piloted by three general practitioners.

In the questionnaire the doctors indicated their knowledge of the patient's medical history on a five point scale, ranging from none to excellent. Resources evaluated were: use of laboratory including $x$ ray examinations, expectant management, prescriptions, sickness certification, referrals to other parts of the health care system, and consultation time. For all these except consultation time doctors indicated whether they had considered using each resource. If so, they had to record whether they felt the decision was $(a)$ taken on the basis of the clinical presentation alone, including history and findings, with previous knowledge about the patient playing little or no part; $(b)$ influenced by some previous knowledge, causing them to use more or less of the resource; or $(c)$ influenced by lack of previous knowledge, causing more or less use of the resource. The last part of each of the five questions recorded whether the resource had actually been used. Expectant management was assessed by the doctor. The doctor also estimated how previous knowledge influenced the consultation time. "Considerably more or less time" was defined as $50 \%$ more or less time than would otherwise have been used, "somewhat more or less" as between $20 \%$ and $50 \%$. The doctors were encouraged to write additional comments for each answer.

The reason for the consultation was classified as new, follow up (for medical problems that first presented less than six months ago), chronic (problems lasting six months or longer or flare ups of a chronic disease), and preventive (including maternity care and general health checks carried out as part of the doctor's 
TABLE I-Influence of previous knowledge about the patient on time spent in the consultation. Results are numbers (percentages)

\begin{tabular}{lcccccc}
\hline & \multicolumn{5}{c}{ Time spent in the consultation } & \\
\cline { 2 - 6 } Previous knowledge & Much more & $\begin{array}{c}\text { Somewhat } \\
\text { more }\end{array}$ & $\begin{array}{c}\text { Little or no } \\
\text { influence }\end{array}$ & Somewhat less & Much less & Total \\
\hline None & $44(11)$ & $85(22)$ & $264(67)$ & $0(0)$ & $0(0)$ & $393(100)$ \\
Slight & $24(3)$ & $126(14)$ & $519(56)$ & $223(24)$ & $28(3)$ & $920(100)$ \\
Some & $28(3)$ & $110(10)$ & $429(40)$ & $420(40)$ & $77(7)$ & $1064(100)$ \\
Good & $28(3)$ & $103(10)$ & $304(30)$ & $435(43)$ & $154(15)$ & $1024(100)$ \\
Excellent & $21(4)$ & $56(11)$ & $104(20)$ & $202(39)$ & $132(26)$ & $515(100)$ \\
\hline Total & $145(4)$ & $480(12)$ & $1620(41)$ & $1280(33)$ & $391(10)$ & $3916(100)^{\star}$ \\
\hline
\end{tabular}

$\star 2$ Cases missing.

TABLE II - Influence of previous knowledge on time spent in the 2296 consultations where the presence or absence of previous knowledge did play a part ${ }^{\star}$

\begin{tabular}{|c|c|c|c|c|}
\hline $\begin{array}{l}\text { Use of time } \\
(0=\operatorname{less}(n=1671), 1=\text { more }(n=625))\end{array}$ & No & Odds ratio $(95 \% \mathrm{CI})$ & Estimate & SE \\
\hline \multicolumn{5}{|l|}{ Previous knowledge: } \\
\hline None & 129 & $1 \cdot 0$ & & \\
\hline Slight & 401 & $0.073(0.06$ to 0.13$)$ & $-2 \cdot 62$ & $0 \cdot 30$ \\
\hline Some & 635 & $0.033(0.02$ to 0.05$)$ & $-3 \cdot 41$ & $0 \cdot 30$ \\
\hline Good & 720 & $0.027(0.01$ to 0.05$)$ & $-3 \cdot 60$ & 0.30 \\
\hline Excellent & 411 & $0.028(0.02$ to 0.05$)$ & $-3 \cdot 58$ & 0.31 \\
\hline \multicolumn{5}{|l|}{ Sex: } \\
\hline Male & 818 & $1 \cdot 0$ & & \\
\hline Female & 1478 & $1.57(1.26$ to 1.96$)$ & 0.45 & $0 \cdot 11$ \\
\hline \multicolumn{5}{|l|}{ Age (years): } \\
\hline$\leqslant 15$ & 93 & $1 \cdot 0$ & & \\
\hline $16-69$ & 1691 & $2.03(1.13$ to 3.64$)$ & 0.71 & 0.30 \\
\hline$\geqslant 70$ & 512 & $1.43(0.77$ to 2.66$)$ & $0 \cdot 36$ & 0.32 \\
\hline \multicolumn{5}{|l|}{ Type of illness: } \\
\hline New & 586 & $1 \cdot 0$ & & \\
\hline Follow up & 597 & $0.52(0.39$ to 0.69$)$ & -0.65 & $0 \cdot 14$ \\
\hline Chronic & 904 & $0.64(0.50$ to 0.83$)$ & $-0 \cdot 44$ & $0 \cdot 13$ \\
\hline Preventive care & 209 & $0.40(0.25$ to 0.56$)$ & $-1 \cdot 00$ & 0.21 \\
\hline
\end{tabular}

*Odds ratios were calculated to control for the effects of sex, age, and type of illness. For each factor odds ratios are expressed relative to a baseline comparison group. When the $95 \%$ confidence intervals do not include 1 the odds ratios are signifcantly different at the $5 \%$ level. regular surgery). The main reason for the consultation was noted by the doctor and subsequently coded by the authors in accordance with the new International Classification of Primary Care, ICPC. ${ }^{5}$

In the first statistical analysis the dependent variable time was dichotomised into using more or less time than the doctor would otherwise have spent. Knowledge about the patient's previous medical history, the major independent variable, was held at each level of the five point scale. In the second analysis the dependent variables were dichotomised into using more or less of the resource in question. Previous knowledge was dichotomised into scant or good, based on the doctor's indication that previous knowledge, or lack of it, influenced the use of the resource.

The patients were divided into three age groups: $0-15,16-69$, and 70 and older.

When logistic regression was used the estimates were transformed into odds ratios by exponentiating the regression estimates to assess the relative importance of each of the factors evaluated. ${ }^{6}$

\section{Results}

All but two of the 133 general practitioners who had agreed to take part in the study actually participated. Of 3990 possible records $3918(98 \%)$ were returned. The use of resources and the patients' age, sex, and morbidity patterns were closely compatible with those in previous surveys of Norwegian general practice. ${ }^{78}$

Among the 3918 consultations 1417 were due to new medical problems, 911 for follow up, 1184 for chronic problems, and 406 for preventive or administrative reasons.

TIME

The doctor's knowledge about the patient's medical history influenced the time spent in $59 \%$ of all consultations, in $16 \%$ causing more time to be spent and in $43 \%$ less (table I). In the $3523(90 \%)$ of consultations where the doctor had previous knowledge, time was saved in $47 \%$ and prolonged in $14 \%$. Among the 49 consultations where the doctor indicated good or excellent previous knowledge but still used much more time, 21 were for psychiatric or social problems. The frequency of such problems among all patients where the doctor had good or excellent knowledge was $10 \%$. Of the 391 consultations where much time was saved due to previous knowledge, 242 were related to musculoskeletal, cardiovascular, or psychiatric problems. Previous knowledge had least influence on use of time in consultations related to somatic problems such as ear, eye, or skin infections.

- In the 370 consultations for new medical problems and where the doctor had good or excellent knowledge of the patient, this knowledge was thought to save time in 163 of the consultations, while prolonging it in 19 . Among the 296 encounters due to new problems and where the doctor lacked any previous knowledge this lack of information was considered a hindrance in 89 .

After controlling for age, sex, and type of illness, we concluded that significant time was saved with increasing previous knowledge about the patient (table II).

\section{THE LABORATORY}

Laboratory tests were the resource most often considered, and used, in the consultation (table III). Previous knowledge influenced $43 \%$ of all decisions on how to use the laboratory; lack of knowledge influenced $7 \%$. In consultations where the presence or absence of previous knowledge did play a part, laboratory tests were more than 10 times more likely to be ordered when previous knowledge was scant, controlled for the effects of gender, age, and type of illness (table IV).

\section{EXPECTANT MANAGEMENT}

Expectant management was considered in $33 \%$ of all consultations. Of these decisions $65 \%$ were influenced by the presence or absence of previous knowledge (table III). In $82 \%$ of the cases where the doctor indicated good previous knowledge a wait and see policy was used more freely. When the doctor was influenced by inadequate previous knowledge it was used more restrictively in $43 \%$. With good previous knowledge the doctor was three times more likely to manage expectantly than with scant knowledge, controlled for the other independent variables (table IV).

\section{PRESCRIBING}

A prescription was considered in half of all consultations (table III), and $63 \%$ of these decisions were taken on the basis of the current clinical presentation alone. Previous knowledge influenced the doctor to prescribe more liberally in $42 \%$ of the consultations and more restrictively in $58 \%$. When the doctor lacked previous knowledge the prescribing practice was more restrictive in $75 \%$ of the cases. Among these patients $41 \%$ had musculoskeletal and $23 \%$ had psychological problems, compared with $19 \%$ and $8 \%$ among patients in general being considered for medication. In 17 cases doctors felt they prescribed more than they would have done with a better knowledge of the patient's medical history; 10 of these consultations were for ear or skin infections.

After the patient's age, sex, and type of illness had been controlled for, prescriptions were the resource least influenced by previous knowledge (table IV). With good previous knowledge the doctors were twice as likely to prescribe than when they had scant previous knowledge.

\section{SICKNESS CERTIFICATION}

Sickness certification was considered in $21 \%$ of all encounters, or in $24 \%$ of patients $16-69$ years of age.

.




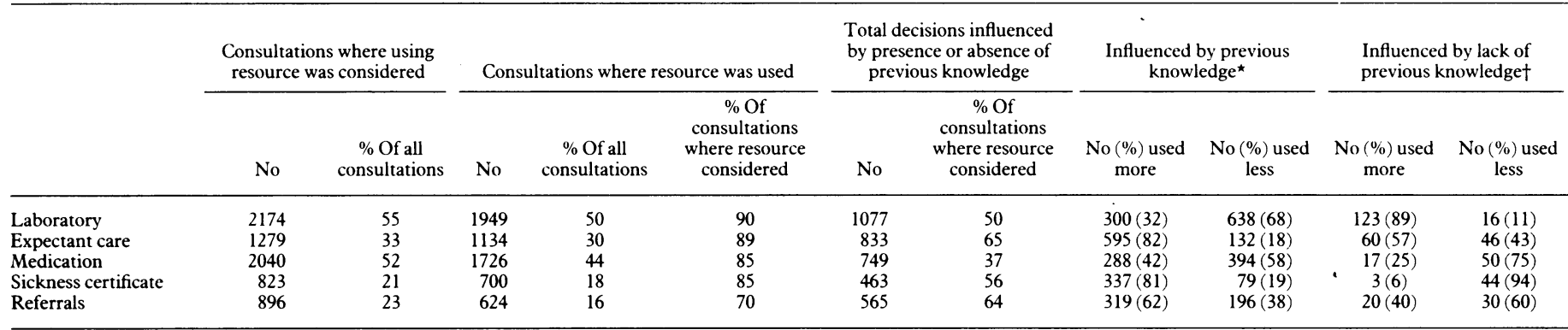

$\star$ Percentages are of total influenced by presence of knowledge.

†Percentage are of total influenced by absence of knowledge.

TABLE IV-Influence of accumulated knowledge on use of resources in consultation, controlled for patient's age, sex, and type of illness $\star$

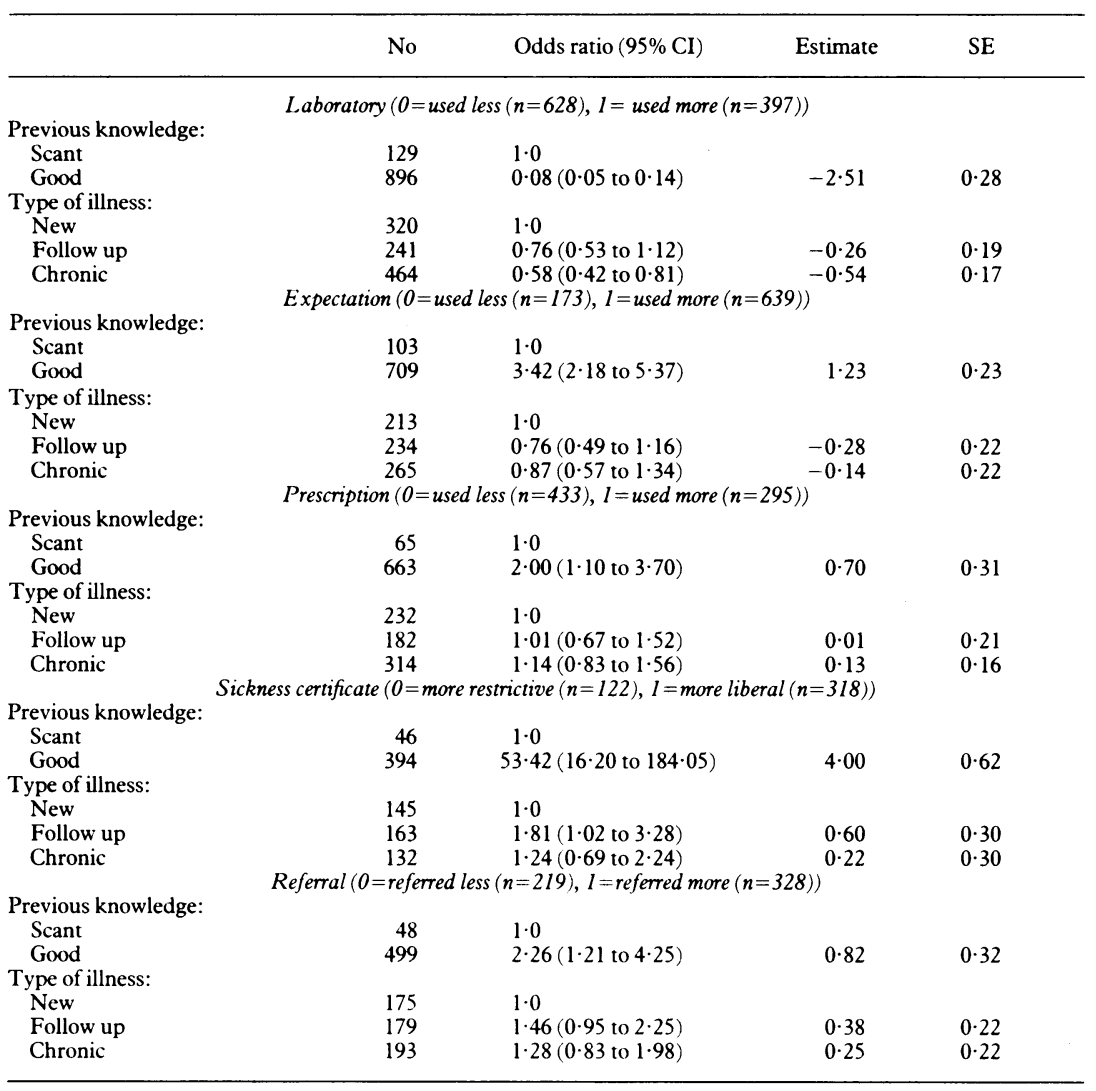

* Odds ratios were calculated to control for the effects of sex, age, and type of illness in each of the five groups of resources. For each factor odds ratios are expressed relative to a baseline comparison group. When the $95 \%$ confidence intervals do no include 1 the odds ratios are significantly different at the $5 \%$ level. The values for age and sex are not shown in the table as none were significant at the $5 \%$ level. Consultations due to preventive or administrative reasons were not included in this analysis as the resources in question are seldom used in these encounters.

The decision whether or not to issue a sickness certificate was influenced by previous knowledge in $56 \%$ of the cases (table III). When controlling for the other independent variables, sickness certification was the resource most often influenced by previous knowledge (table IV). Patients had a 53 times greater chance of getting a sickness certificate if the doctor knew them well than if he did not.

\section{REFERRALS}

Referral was the least used resource (table III). The decision whether to refer was, however, influenced by the presence or absence of previous knowledge in $64 \%$ of decisions. Among patients of whom the doctor had good knowledge $62 \%$ were referred more readily. In $60 \%$ of those of whom the doctor knew little, the doctors were more restrictive with referrals. Patients had a statistically significant, twofold increased chance of being referred if the doctors knew their medical history (table IV).

\section{REASONS FOR CONSULTATION}

Table $\mathrm{V}$ shows the effect of knowledge in consultations for new medical problems where doctors made new diagnostic or management decisions.

\section{Discussion}

This study is largely based on subjective evaluations. The key independent variable was the doctors' reported knowledge about the patient. Did they know what they said they did? Previous studies have indicated that doctors have scant factual knowledge about their patients' life problems or family history..$^{910}$ Factual knowledge is, however, just part of the wider knowledge accumulated in a patient-doctor relationship, a knowledge often used subconsciously. ${ }^{12}$ Such tacit knowledge is difficult to evaluate. Psychometric testing indicates that subjective rating scales of subjects' own functions have good validity, usually better than registration of objective factors alone. ${ }^{1314}$ Thus it is reasonable to accept the doctor's own evaluation as a valid indicator of his or her general knowledge of each patient.

The doctors indicated whether they felt that the presence or absence of previous knowledge influenced the use of resources. This reasoning process may be difficult in itself, possibly yielding answers of low reliability. Each evaluation, however, was related to a very recent consultation, and this close linkage should have increased the reliability and validity of the reasoning. Efforts were made to increase reliability: the questions were carefully worded, evaluated by an expert panel, and pretested and discussed with a group of general practitioners. A two page questionnaire guide, with examples, was provided to all participants. Ninety eight per cent of all questionnaires were returned, and less than five per cent were incomplete or incorrectly marked. No major misunderstandings or discrepancies were uncovered in personal communications with the participants.

A previous evaluation among the participating doctors indicated that they valued continuity of care,${ }^{15}$ so they may have overestimated the impact of accumulated knowledge on the use of resources. In previous studies similar biases had less impact than expected, ${ }^{16} 17$ but they should be kept in mind when evaluating our data.

\section{USE OF TIME}

When the doctor knew the patient time was saved in more than $40 \%$ of all consultations - and considerable amounts of time in one in ten. Conversely, in consultations where the doctors had no previous information one third of consultations were felt to be prolonged owing to lack of knowledge, $11 \%$ substantially so. Lack of previous knowledge was especially a hindrance among patients with psychosocial problems.

There was no linear relation between the level of previous knowledge and the likelihood of saving time. The odds ratio rose sharply between no and slight previous knowledge and levelled off between some and good previous knowledge (table II). It appeared that the level of previous knowledge reached a saturation point where a further increase had little or no effect on time spent in the consultation. A previous analysis has shown that accumulation of knowledge is linked to the duration and depth of the doctor-patient 
TABLE V-Influence of accumulated knowledge on use of resources related to new medical problems, controlled for the patient's age and sex ${ }^{\star}$

\begin{tabular}{|c|c|c|c|c|}
\hline & No & Odds ratio $(95 \% \mathrm{CI})$ & Estimate & SE \\
\hline \multicolumn{5}{|c|}{ Laboratory $(0=$ used less $(n=147), l=$ used more $(n=173))$} \\
\hline Previous knowledge: & & & & \\
\hline Scant & 96 & 1.0 & & \\
\hline \multirow{2}{*}{\multicolumn{5}{|c|}{ Expectant management $(0=$ used less $(n=68), l=$ used more $(n=245))$}} \\
\hline & & & & \\
\hline Previous knowledge: & & & & \\
\hline Scant & 71 & $1 \cdot 0$ & & \\
\hline Good & 242 & $2.77(1.54$ to 5.00$)$ & $1 \cdot 02$ & $\cdot 30$ \\
\hline \multicolumn{5}{|c|}{ Prescription $(0=$ used less $(n=88), 1=$ used more $(n=34))$} \\
\hline Previous knowledge: & & & & \\
\hline Scant & 34 & $1 \cdot 0$ & & \\
\hline Good & 88 & $1.31(0.46$ to 3.77$)$ & $0 \cdot 27$ & .54 \\
\hline \multicolumn{5}{|l|}{ Use of expectation: } \\
\hline No & 100 & $1 \cdot 0$ & & \\
\hline Yes & 22 & $0 \cdot 10(0.03$ to $0 \cdot 28)$ & $-2 \cdot 35$ & .55 \\
\hline \multicolumn{5}{|c|}{ Sickness certificate $(0=$ more restrictive $(n=60), I=$ more liberal $(n=85))$} \\
\hline Previous knowledge: & & & & \\
\hline Scant & 34 & 1.0 & & \\
\hline \multirow{2}{*}{\multicolumn{5}{|c|}{ Referral $(0=$ referred less $(n=82), l=$ referred more $(n=93))$}} \\
\hline & & & & \\
\hline Previous knowledge: & & & & \\
\hline Scant & 30 & 1.0 & & \\
\hline Good & 145 & $3 \cdot 25(1.37$ to $7 \cdot 71)$ & $1 \cdot 18$ & .44 \\
\hline
\end{tabular}

*Odds ratios were calculated to control for the effects of sex and age in each of the five groups age in each of the five groups
where resources were used. The where resources were used. The
odds ratios are expressed relative to a baseline comparison group. When the $95 \%$ confidence intervals do not include 1 the odds ratios are signifcantly different at the $5 \%$ level. The values for age and sex are not shown in the table as none were significan at the $5 \%$ level.

relationship (P Hjortdahl, unpublished study). In Norway it took an average of one to five years, or four or five consultations within 12 months, for the doctors to develop a moderate knowledge base. The first years of a doctor-patient relationship thus seem to have the major impact on accumulated knowledge and the use of time in the consultation.

Furthermore, the relation between knowledge and time was bidirectional. In $14 \%$ of the 1539 consultations where the doctor indicated good or excellent previous knowledge more time was used. This may appear illogical. From interviews with doctors having used this option, however, it became evident that this usually happened when, owing to familiarity between doctor and patient, several nonrelated problems were raised and dealt with concomitantly. Others felt that consultations were prolonged by social chatter. As expressed by one doctor: "I know this lady well. When she is here to check her hypertension, we usually also talk about her husband and their adult children. This social talk takes extra time, but we both enjoy it, and I feel this added information may save time later." This partly reflects the difficulty of doing a cross sectional study on a longitudinal phenomenon. Because the doctor knows the patient he or she may use extra time, or other resources, in one consultation and save doing so later. It also reflects difficulties that may arise in close doctorpatient relationships, where use of resources may be based on old habits rather than sound clinical reasoning. ${ }^{18}$

\section{OTHER RESOURCES}

All resources evaluated were influenced by previous knowledge, although to a varying degree. Accumulated knowledge led to fewer tests, more use of expectant management, slightly less use of prescriptions, and more ready use of sickness certification and referrals. Conversely, lack of knowledge caused a relative increase in the use of tests and less use of a wait and see policy, prescriptions, sickness certification, and referrals.

The decisions whether or not to wait, refer, or certify were most sensitive to previous knowledge; more than $60 \%$ of these decisions were thus influenced. The total impact of accumulated knowledge on resource use also depends on how often the resource is used. Laboratory tests, prescriptions, and expectant management were in absolute numbers the resources most often influenced by the presence or absence of previous knowledge.

As with time, previous knowledge influenced the use of resources in both directions. This is especially evident in use of medication, where previous knowledge caused the doctors to be more liberal with medication in $42 \%$ of the decisions and more restrictive in $58 \%$. Previous knowledge played a part in more than $30 \%$ of all decisions whether or not to use medication, but caused an overall decrease of only $5 \%$ in actual us ?. The total use of a resource is not necessarily an indicator of good medical care.

Expectant management is a recognised diagnostic and therapeutic tool of general practice. ${ }^{19}$ Expectant management may be linked to continuity of care and accumulated knowledge in two different ways. With background information the doctor may find it clinically safe to wait and see rather than prescribe or refer right away. Secondly, by managing expectantly the doctor implicitly takes on a willingness to see the patient again.

In consultations for new medical problems the use of laboratory services, prescribing, and referrals may be inversely related to the use of expectant management. When use of a wait and see policy was included in the regression analysis between use of laboratory tests and previous knowledge and between referrals and previous knowledge the strength of association was to some degree reduced, but not significantly so. When consultations due to new medical problems were controlled for the use of expectant management previous knowledge was no longer significantly associated with prescribing. When expectant management was used in these consultations there was a significant, tenfold reduced chance of prescribing.

Expectant management was considered used in one third of all consultations and was the resource most often influenced by previous knowledge. This indicates that general practitioners actively use expectation, which in turn influences them to use less of other resources.

In consultations for new medical problems, after controlling for age and sex of the patient, we found that previous knowledge reduced greatly the likelihood of using laboratory tests, increased the likelihood of writing sickness certificates, and significantly but modestly increased the likelihood of using expectant management and referrals. The doctor's prescription pattern was the resource least influenced by previous knowledge.

1 McWhinnev IR. Continuity of care. $\mathcal{F}$ Fam Pract 1982;15:847-8.

2 Sheldon M, Brooke J, Rector A. Decision-making in general practice. London: Macmillan, 1985.

3 Council on Long Range Planning and Development. The future of family practice. Implications of the changing environment of medicinc. $\mathcal{F} A M A$ $1988 ; 260: 1272-9$

4 Secretaries of State for Health, Wales, Northern Ireland, and Scotland. Wiorking for patients. London: HMSO, 1989.

5 Bentsen BG. International classification of primary care. Scand J Primary Health Care 1986:4:43-50.

6 Hosmer DW, Lemenshow S. Applied logistic regression. New York: Wiley, 1989.

7 Rutle O. Pasienten fram i lyset-Analyse av legekontaktar i primarhelsetenesta [Getting the patient into the limelight. An analysis of encounters in primary [Getting the patient into the limelight. An analysis of encounters in primary

8 Nylenna M. Why do our patients see us? Scand 7 Primary Health Care $1985 ; 3: 155-62$.

9 Yaffe $M \mathrm{~J}$, Stewart MA. Factors influencing doctors' awareness of the life problems of middle-aged patients. Med Care 1985;23:1276-82.

10 Rosenberg EE, Pless IB. Clinicians' knowledge about the families of their patients. Family Practice 1985:2:23-9.

11 Polanyi M. The tacit dimension. New York: Doubleday, 1967

12 Schön DA. The reflective practitioner. How professionals think in action. New York: Basic Books, 1983

13 Mossey JM, Shapiro E. Self-rated health: a predictor of mortality among the elderly. Am f Publ Health 1982;72:800-8.

14 Andrews FM. Construct validity and error components of survey measures: a structural modeling approach. Public Opinion Quarterly 1984;48:409-42.

15 Hortdahl P. Ideology and reality of continuity of care. Fam Med 1990;22: $361-4$.

16 Cartwright A, Lucas S, O'Brian M. Some methodological problems in studying consultations in general practice. $\mathcal{J} R$ Coll Gen Pract 1976;26: 895.906 .

17 Nylenna M. Open prospective recording: how is the doctor influenced? Family Practice 1986;3:240-5.

18 O'Dowd TC. Five vears of heartsink patients in general practice. BMJ 1988.297:528-30.

19 McWhinnev IR. A textbook of family medicine. New York: Oxford University Press, 1989

(Accepted 30 July 1991 